\title{
Le langage à l'école pour apprendre, se construire, devenir un citoyen
}

\section{Dominique Bucheton}

\section{(2) OpenEdition \\ 1 Journals}

\section{Édition électronique}

URL : http://journals.openedition.org/trema/1740

DOI : 10.4000/trema. 1740

ISSN : 2107-0997

\section{Éditeur}

Faculté d'Éducation de l'université de Montpellier

\section{Édition imprimée}

Date de publication : 1 octobre 1999

Pagination : 103-114

ISSN : 1167-315X

\section{Référence électronique}

Dominique Bucheton, «Le langage à l'école pour apprendre, se construire, devenir un citoyen », Tréma [En ligne], 15-16 | 1999, mis en ligne le 01 octobre 1999, consulté le 30 avril 2019. URL : http:// journals.openedition.org/trema/1740 ; DOI : 10.4000/trema.1740

Ce document a été généré automatiquement le 30 avril 2019.

Trema 


\title{
Le langage à l'école pour apprendre, se construire, devenir un citoyen
}

\author{
Dominique Bucheton
}

\section{Présentation de l'atelier}

1 Il ne peut y avoir de citoyenneté possible sans la construction de la singularité de la pensée. L'écriture, la lecture et l'oral pensés comme des pratiques réflexives d'un sujet permettent de construire un rapport autonome au savoir et au pouvoir conféré par le savoir. L'école doit les développer davantage. Elles donnent ainsi aux élèves le pouvoir de penser et le pouvoir de réfléchir par eux-mêmes. Il ne peut y avoir de citoyenneté possible sans la construction de cette singularité de la pensée. En même temps, il ne peut y avoir de pensée singulière sans la confrontation, la négociation avec l'autre. Toujours singulière, la parole est en même temps dialogue. Un dialogue qui est le fondement de l'action commune, de la vie sociale, de la démocratie. Langage, savoir, identité et citoyenneté sont donc des concepts que l'école doit apprendre à davantage conjuguer ensemble.

2 L'objectif est de promouvoir dans la classe, en formation, un espace de parole orale et écrite qui permette la délibération, le questionnement, l'invention, la construction en commun de savoirs et de valeurs. Construction forcément singulière, instable, qui s'inscrit dans le développement de personnes en devenir.

3 Mais cette conception des apprentissages langagiers se heurte à des difficultés très diverses. Elle demande de profondes modifications dans les pratiques de formation, d'enseignement et notamment dans les évaluations. C'est à l'analyse de ces difficultés ainsi qu'à l'élaboration de propositions qu'ont contribué les intervenants que nous avons invités dans l'atelier pour engager le débat avec la salle.

4 Nous avons donc demandé à divers praticiens et chercheurs de proposer des pistes de réflexion et de débat: à partir de pratiques dans des classes, (Bruno Seweryn et Alain Dunas: IMF sur le site IUFM de Perpignan); à partir d'expérimentations dans le cadre 
d'une recherche sur le débat philosophique en primaire, (M. Tozzi,université P. Valéry); et pour terminer, sur la trace, dans les mémoires professionnels, des conceptions de la citoyenneté chez les jeunes enseignants (R. Etienne, IUFM Montpellier, équipe Esterel).

\section{Alain Dunas. Témoignages de l'équipe de l'Ecole Biaise Pascal de Perpignan}

\section{Quelles situations mettre en place pour favoriser des pratiques langagières réflexives et sociales?}

L'école B. Pascal est en grande majorité fréquentée par les enfants de la cité voisine dont la population est composée pour une très large part de familles marocaines mais aussi de quelques familles algériennes, turques et françaises. Ces familles, touchées par le chômage et la précarité ont un niveau social assez bas.

6 Ce qui caractérise d'abord nos élèves est un niveau scolaire relativement bas dû principalement à une mauvaise maîtrise de la langue française écrite et une pratique de la langue orale pauvre (utilisation d'un vocabulaire minimum, emploi de mot-phrases...) ou stérile (beaucoup de répétitions narratives peu enrichissantes).

7 Par ailleurs, ces élèves ont à gérer de nombreux conflits intérieurs. Issus de cultures différentes, ils sont confrontés à une institution scolaire par rapport à laquelle ils ont du mal à se situer et faute de repères, ont du mal à se constituer une identité. La représentation qu'ils ont de l'école et d'eux-mêmes étant souvent négative, les apprentissages ont peu de sens à leurs yeux.

D'où l'intérêt de la réflexion menée depuis deux ans avec les membres de l'équipe de recherche DIDAXIS (J.C. Chabanne et D. Bucheton) qui nous a amenés, certains de mes collègues et moi-même, à modifier nos pratiques de classe sur la base de trois hypothèses :

- le désir et le plaisir d'apprendre n'apparaissent que lorsque l'élève a de lui même une image singulière et positive ;

- les élèves et les savoirs se co-construisent ;

- l'écrit et l'oral sont les piliers de cette co-construction.

9 L'enjeu est donc d'aider les élèves à se construire une identité au moins scolaire. Pour ce faire, chacun d'entre nous expérimente des pratiques orales et écrites réflexives de la langue en fonction de la spécificité de la classe et des savoirs enseignés. Les "outils" utilisés sont le "cahier de travail », le «cahier d'écriture », la " correspondance intragroupe », ou encore le « conseil de classe ».

Dans ma classe, j'observe des changements significatifs de comportement chez certains élèves depuis que nous utilisons ces « outils».

\section{Le cahier de travail}

11 Sur ce cahier, les élèves posent les représentations qu'ils ont de la notion qui va être abordée (ce que je pense...), ils peuvent aussi décrire la démarche utilisée pour effectuer le travail demandé (comment je fais...) et, en fin de séance, notent ce qu'ils ont appris en regard de leur formulation initiale. L'élève mesure ainsi le chemin parcouru dans ses 
apprentissages. Par ailleurs, les cahiers pouvant être échangés, ils sont confrontés à des idées différentes... On note ainsi des progrès dans les contenus et les formes des énoncés (termes du vocabulaire propres à la notion étudiée, phrases plus élaborées). En écrivant, ces élèves intègrent mieux certains savoirs et prennent conscience qu'ils sont capables de les formuler eux-mêmes.

12 Cette pratique d'un écrit « de travail », ni corrigé, ni noté, libère l'élève des contraintes cognitives liées à l'orthographe et la mise en forme (travaillées dans d'autres situations), et sert de support à un oral réflexif avec les camarades. L'étape préalable de l'écriture individuelle aide à l'organisation de la pensée et à la formulation des propos. Elle oblige chaque élève à être acteur de la parole collective qui sera ensuite élaborée. Elle prépare chacun à assumer un point de vue singulier.

\section{Le cahier d'écriture}

13 On y travaille par des séries de consignes et de lanceurs successifs pour faire évoluer les textes courts ou longs, le plus souvent de fiction. Dans un premier temps, un travail sur « l'épaisseur» du texte, par réécritures successives, doit amener progressivement les élèves à modifier leur posture d'écriture. Ensuite, un travail sur la surface du texte va justifier les activités de structuration des notions grammaticales, d'orthographe de conjugaison, de vocabulaire. Ces activités de réécriture favorisent la mise à distance de la chose écrite. Questionner l'écrit, son propre écrit, nous semble une attitude essentielle à mettre en place.

\section{La correspondance intra-groupe}

14 Cette pratique de l'écrit qui permet aux élèves de s'envoyer des messages (chaque enfant possède sa boîte aux lettres), observable dans deux classes, repose sur le principe que le rapport au savoir se construit aussi dans le rapport et le dialogue avec l'autre. En envoyant et en recevant un message écrit et pensé, l'élève se situe dans une réalité sociale dont il est un acteur singulier. Ce réseau d'échanges écrits permet à la fois de satisfaire les interrogations que se posent les élèves les uns par rapport aux autres et de réguler les conflits. La correspondance a fait disparaître pratiquement toutes violences physiques et verbales dans la classe. Ces élèves pratiquent ce type d'échange depuis le CE1. La fréquence des messages ne baisse pas.

\section{Le conseil de classe}

15 Cette réunion hebdomadaire permet la délibération, l'échange de points de vue et l'argumentation. Quand un conflit persiste nous en débattons. Le conseil de classe, au delà de sa fonction de régulation des problèmes de la classe, doit amener l'élève à prendre conscience que son propos est singulier mais que d'autres peuvent penser différemment ; ce lieu de construction de la citoyenneté apparaît réellement comme un espace de paroles dans lequel se discute la légitimité de chacun à revendiquer des droits et à reconnaître ses devoirs.

Pour conclure, il semblerait que ces pratiques permettent à une majorité de nos élèves de mieux saisir le sens des tâches que nous leur proposons, d'avoir de la langue orale et écrite une autre perception. Donc des signes encourageants de réussite chez certains. 
Pour autant, il nous reste de nombreuses zones d'ombre à éclaircir : celle de la lisibilité de nos démarches par les familles de la cité, par l'ensemble des collègues ou par l'institution elle-même dans sa dimension hiérarchique.

Il est difficile encore en effet au sein même de l'école d'expliquer aux collègues qui ne sont pas associés à notre travail qu'une pratique de l'écrit pour penser ne s'oppose pas à un travail de structuration de la langue.

Difficile aussi d'évaluer le travail d'élaboration dans lequel s'opère la construction du sujet et de ses savoirs. Par rapport aux exigences normées que constituent les évaluations nationales, les effets de notre travail pèsent peu sur les résultats. Même si les évaluations de CE2 de cette année montrent une amélioration globale du score moyen en français, par rapport à l'année dernière, les résultats restent en dessous de la moyenne nationale. Pour autant, nous ne voulons pas être les « vilains petits canards » de la circonscription et nous souhaiterions plus de liaison entre le «terrain » et nos supérieurs hiérarchiques. Un vrai " contrat de réussite ", négocié sur la base de la réalité des élèves et des problèmes à traiter d'urgence. La gestion démocratique et citoyenne de la politique des établissements reste à inventer.

\section{Bruno Seweryn. Trois ans d'expérimentation, école Neffiach}

\section{Faire écrire - penser les élèves au quotidien : en quoi cela contribue-t-il à la construction du sujet singulier et social ?}

Comme le précise Philippe Meirieu ${ }^{1}$, «pour Jules Ferry, la lecture et l'écriture étaient le moyen de libérer le peuple de toutes ses chaines. Aujourd'hui, lire et écrire sont devenus pour des milliers d'enfants, une obligation fastidieuse et le début de l'échec. »

Il s'agit de privilégier d'autres formes où l'enfant est réellement au centre de son écrit : cahier d'expérimentation (Charpak - Main à la pâte), écrits épistolaires, cahiers d'écrivain que j'ai appelé « cahiers d'écrits quotidiens ».

21 Description rapide du cahier d'écrivain : c'est un cahier où les élèves écrivent 3 textes par semaine à partir de lanceurs (des thèmes, des sujets). Ceux-ci sont imposés, seminégociés ou négociés. L'échange est effectué sous forme d'inter-lectures, d'oralisation spontanée ou choisie par les camarades. Les commentaires sont faits dans une perspective constructive au sein d'un espace de discussion. Le discours de l'enfant s'inscrit donc ici comme maillon dans la chaîne verbale puisqu'il réfère à ce qui a existé et engendre d'autres discours (M. Bakhtine). Il est donc plus vrai que pour une seule évaluation du maître.

Le discours de l'enfant s'inscrit donc ici de façon dynamique puisqu'il réfère à ce qui a existé et engendre d'autres discours : c'est un élément d'« une chaîne verbale ininterrompue ${ }^{2}$ ». Le lire, l'écrire et le parler sont ici étroitement liés. C'est aussi un espace de parole partagée et d'exploration ludique du langage mais qui est protégé par la classe : les écrits ne sont pas communiqués au dehors, on peut choisir de lire ou de ne pas lire à la classe, on peut choisir de ne pas écrire à partir d'un lanceur en utilisant un joker. 


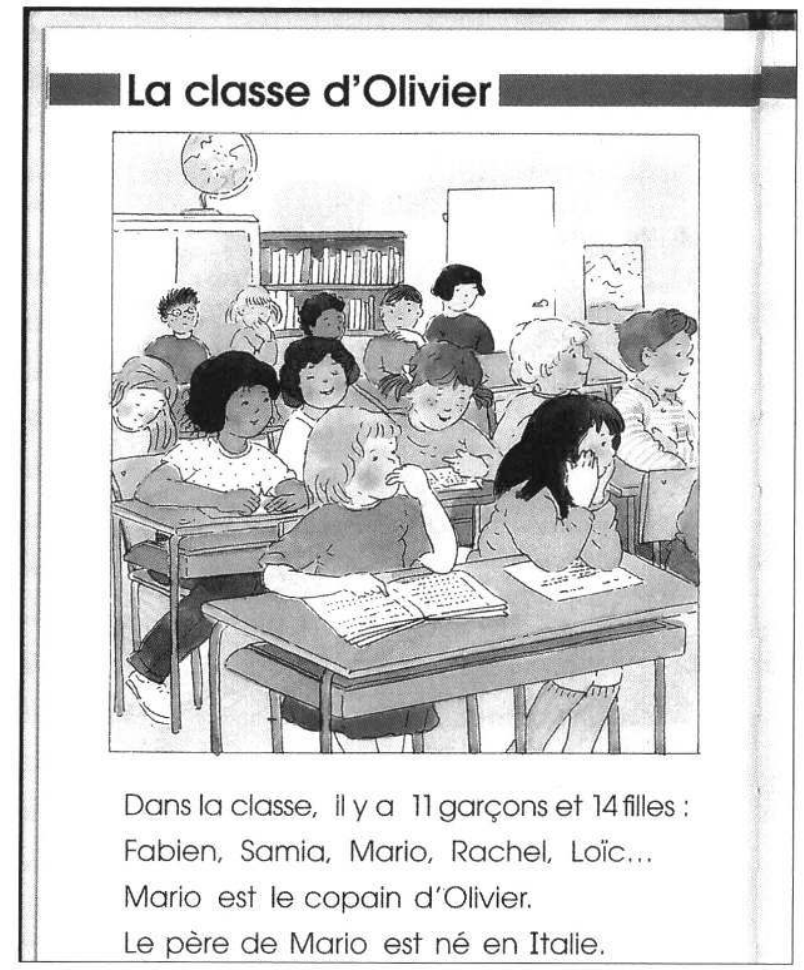

VIAN P., BONNEVIE P. (et al.) : Lecture en fête - CP, Livre 1(Collection : Hachette Éducation). Paris, Hachette, 1993, 96 p., p. 18. Illustrations : Joëlle BOUCHER.

Certains lanceurs sont plus moteurs d'écrits et facteurs de progrès que d'autres.

Ils améliorent la qualité de ce qui est écrit, la renforcent dans la variété de types de séquences textuelles, la portée symbolique, l'épaisseur sémantique. Les enfants se transforment progressivement, leurs postures d'écriture bougent.

\section{Les constats}

\section{Sur le plan linguistique}

L'orthographe est spontanément mieux maîtrisée dans les premiers jets (de $12 \%$ à $6 \%$ pour l'élève le plus fort, de $60 \%$ à $30 \%$ d'erreurs pour le plus faible), la conjugaison également et en plus elle se complexifie (temps, modes). La segmentation du texte en paragraphes et phrases est meilleure et les thèmes traités sont mieux agencés selon des progressions linéaires ou éclatées là ou la progression se faisait à thème constant (Cf., Combettes).

Les propos sont plus logiquement enchaînés, les récits s'enrichissent tant au niveau du nombre d'épreuves qu'au point de vue de la polyphonie énonciative.

Des savoirs métalinguistiques se développent conjointement à ces savoirs épilinguistiques.

\section{Sur le plan socio-langagier}

Le contenu des textes s'améliore progressivement mais individuellement et les lanceurs sont choisis selon une séquence implicite : proche, général, imaginaire, argumentatif. Ce dernier point est essentiel dans la construction du citoyen. 
Un écart existe entre ce que veut faire l'enfant et ce qu'il peut faire, entre ce qu'il veut faire et ce que l'enseignant le croit capable de faire.

Les parents sont également concernés par ce travail et les enfants commencent à faire des calculs sur les lecteurs potentiels social et le scolaire s'accrochent-ils, notamment au niveau de l'enseignement de la langue ou plutôt de la maîtrise des langages? ».

J'ai ouvert plusieurs portes : la place de l'oral et de la communication dans la production langagière et son influence sur l'écrit, la construction de l'identité chez le sujet écrivant beaucoup, la construction conjointe du récit, de l'argumentation et du développement de l'enfant.

Former un citoyen capable d'agir sur la société n'est donc pas chose facile. Néanmoins, c'est un enjeu majeur de notre système éducatif.

Il faut donner au futur citoyen une arme : la pensée.

Deux moyens de la développer et de l'exprimer : la parole et l'écriture.

\section{Michel Tozzi. Université Paul Valéry}

\section{L'éveil de la pensée réflexive chez l'élève}

La recherche dont il est question part de l'hypothèse de la faisabilité d'une réflexion philosophique chez l'enfant dans le cadre scolaire. Elle est menée dans un contexte innovant (la philosophie n'intervient en France qu'en classe Terminale de lycée; le programme de morale belge vient d'introduire dès la Première, de la philosophie, et la ville de Bruxelles développe dans ses écoles la philosophie pour les enfants), et à un moment où l'éducation à la citoyenneté (re-)devient un axe fort de la mission des enseignants. 
L'oral et l'écrit réflexifs dans la classe ne peuvent s'inscrire dans une perspective de développement de la citoyenneté que si les enseignants reconnaissent à ces deux activités leur contribution à la formation de la femme ou de l'homme dans une cité moderne : écrire - parler pour penser. Autrement dit, leur projet devient d'aider les élèves à construire une attitude singulière qui en fasse des citoyens capables d'élaborer une pensée personnelle. Le rôle du langage dans la construction d'une personnalité unique mais prête à échanger, à partager avec les autres, s'avère primordial. Il m'a donc semblé opportun $^{3}$ de faire une analyse des mémoires professionnels des professeurs d'école stagiaires (PE2) qui ont choisi de traiter ce sujet de la citoyenneté en 1997-1998 sur le site de Montpellier. L'intention de cette lecture est donc de questionner la conception de la citoyenneté dans les productions des stagiaires et des élèves. 
Cette contribution s'appuie sur la lecture de cinq mémoires professionnels soutenus à Montpellier en juin 1998. Le tableau ci-contre renvoie aux documents sur lesquels nous avons travaillé. Nous n'avons été ni le directeur, ni l'assesseur d'aucun de ces travaux.

\begin{tabular}{l|l|l|l}
\hline \multicolumn{3}{l}{ Mémoires professionnels d'enseignants-stagiaires - Analyse } \\
\hline $\begin{array}{l}\text { Nom \& prénom } \\
\text { (initiales) }\end{array}$ & Titre du mémoire & Discipline & Travaux \\
\hline $\mathrm{CP}$ & $\begin{array}{l}\text { L'insertion par le sport: utopie ou réalité? } \\
\text { Une séquence de balle ovale en Z.E.P... }\end{array}$ & E.P.S. & $\begin{array}{l}\text { Grille } \\
\text { d'auto-évaluation }\end{array}$ \\
\hline $\mathrm{OB}$ & La solidarité (cycle 3) & Éducation civique & Lettre \\
\hline $\mathrm{NV}$ & $\begin{array}{l}\text { Éduquer à la citoyenneté. Les droits et les } \\
\text { devoirs dans les classes }\end{array}$ & Non précisée & $\begin{array}{l}\text { Production et } \\
\text { règles de vie }\end{array}$ \\
\hline $\begin{array}{l}\text { MB } \\
\text { GA }\end{array}$ & $\begin{array}{l}\text { Peut-on, grâce à un projet basé sur des } \\
\text { activités physiques artistiques, améliorer la } \\
\text { cohésion du groupe dans une classe à } \\
\text { double et triple niveaux? }\end{array}$ & E.P.S. & $\begin{array}{l}\text { Spectacle et } \\
\text { comptine }\end{array}$ \\
\hline MV & $\begin{array}{l}\text { Comment réussir un éducation à la différence } \\
\text { Éducation civique / } \\
\text { Géographie }\end{array}$ & Dessins et textes \\
\hline
\end{tabular}

\section{Pourquoi travailler la citoyenneté sur des mémoires professionnels de PE2 ?}

Le rôle de l'enseignant dans l'acquisition du langage n'étant plus à souligner, reste à déterminer quelle langue il parle lui-même quand il étudie la classe, s'il privilégie l'individualisme (évaluation traditionnelle) ou s'il entend proposer un développement des compétences collectives (Guy Le Boterf ${ }^{4}$ ). Ce qui revient à déterminer le statut qu'il accorde à la parole orale ou écrite des élèves.

Il est difficile de distinguer la parole professionnelle de la parole personnelle du stagiaire dans des écrits destinés à rapporter une réflexion sur une expérience professionnelle.

Seule des cinq stagiaires concernés, $\mathrm{CP}$ commence par un récit. Elle décrit les difficultés de Khalid. Les autres jouent sur leur projet ou sur un diagnostic global. Cette conception du mémoire professionnel tend à en faire un travail de recherche dans lequel l'implication de l'auteur est mal venue, où il est malséant de se transformer en narrateur ou encore, a fortiori, en personnage singulier. Or, si l'élève est une personne, l'enseignant est aussi une personne, c'est même un personnage dans la pièce soumise aux unités de lieu, de temps et d'action qui se joue dans la classe. Le faire disparaître et gommer son engagement, ne serait-ce que pour des raisons rhétoriques ou liées à l'émergence d'un nouveau type d'écrit me paraît peu "citoyen " 5 . Écrire sur soi dans la classe, dans l'École, au milieu des élèves, des collègues et des parents, n'est-ce pas s'exposer, témoigner ? La disparition derrière un enseignant type me semble à l'opposé de l'effort actuel de personnalisation. Il est vrai, et nous y reviendrons, que la démocratie éprouve quelque peine actuellement à faire preuve de respect pour les personnes. On ne peut dès lors comprendre l'origine de l'actuelle discrétion des stagiaires dans leur engagement personnel, du moins tel que le traduit ou le trahit le mémoire professionnel.

La lecture attentive des cinq mémoires et l'étude informatisée de lemmatisation ${ }^{6}$ permettent de dresser un premier constat sur les valeurs liées à la citoyenneté : sur cinq mémoires étudiés, deux seulement utilisent des dérivés de «démocrat[...]». En revanche, "citoyenneté " apparaît toujours et il est lié à "socialisation, insertion, intégration et cohésion ». Cela ne fait que confirmer deux constats que les travaux les 
plus récents réaffirment: ce n'est pas la démocratie qui est visée principalement dans l'éducation à la citoyenneté, car elle impose des débats et un mode de décision, situation bien gênante pour une École fondant la dissymétrie des pouvoirs sur la dissymétrie des savoirs. Les écrits de B. Defrance ${ }^{7}$ sont éloquents dans ce domaine. En revanche, la contrainte du groupe impose à l'enseignant de construire une cohésion fondée sur ce que J. Gaillard ${ }^{8}$ appelle la vision romaine de la loi et du droit: ce ne sont pas tant leur rédaction collective qui importe que leur respect par le citoyen. Cela n'est pas critiquable en soi mais la réduction de la démarche citoyenne à un dispositif ingénieux pour éprouver la nécessité de règles doit attirer l'attention de tous sur la dilution de l'attitude citoyenne dans une perspective de simple réflexion en commun.

41 Ces remarques m'incitent à proposer un recentrage sur la notion même de citoyenneté telle qu'elle peut et doit figurer dans la communication orale et écrite au sein de la classe et de l'école. Cette attitude revient à moins invoquer la citoyenneté à tout propos et donc hors de propos pour la situer dans sa réelle sphère d'intérêt : les décisions collectives et l'appréciation de l'efficacité d'une pareille conception des rapports sociaux.

\section{Quels propos sur la citoyenneté ?}

Il y a de grandes et graves confusions entre civilité, civisme et citoyenneté. Elles sont entretenues d'ailleurs par les instructions officielles (IO) et encouragées par le discours ambiant. Il faudrait donner un sens solide, « dur ", au terme " citoyen " pour éviter de le dissoudre dans la cohésion (CP), la solidarité ou le développement (OB), voire l'insertion ou l'intégration (CP) ou encore le respect des différences (MV). Seul, NV ne confond pas le concept avec d'autres, même s'il insiste trop sur l'opposition scolaire des droits et des devoirs (p. 4), car il consent à s'interroger sur ce qui détermine la citoyenneté au regard des textes : «Donc, la citoyenneté ou qualité de citoyen que l'on peut aussi définir comme étant la participation, directe ou indirecte, au gouvernement de la cité (Chagnollaud D. ${ }^{9}$ ), n'est pas naturelle mais s'acquiert. » (p. 5).

Sa contribution va plus loin. Elle respecte le programme aujourd'hui confié à l'éducation à la citoyenneté, qui dépasse l'instruction ou l'éducation civiques, en ce sens qu'il faut bien évoquer la perspective d'un apprentissage du droit de cité, d'une appropriation risquée d'un concept pour lequel il faudra une rupture marquée entre l'école, métaphore de la cité, et l'entrée dans la vie politique, la cité réelle. Ce que nous propose également NV (p. 3) :

«Mais comment l'école peut-elle participer à l'éducation à la citoyenneté? D'après Diane Droryt ${ }^{10}$, l'établissement de barrières, de limites, de règles semble être une étape indispensable afin que les enfants puissent les tester, seule façon pour eux de savoir si elles sont valables et durables. Comment définir ces règles si ce n'est en référence à des droits et des devoirs communs à chaque individu de la classe, de l'école?"

Si la théorie de la citoyenneté est bien située sur son axe politique, il faut en venir aux pratiques de classe et d'école, du moins à un discours écrit sur lesdites pratiques.

L'école est un lieu d'interdits. Ainsi l'effort de NV (p. 12) pour obtenir des règles se heurte-t-il à une capacité plus grande à inventer des devoirs qu'à imaginer les droits les sous-tendant (déclarations des droits et non des devoirs) :

"Si l'on compte l'ensemble des règles des quatre groupes, on arrive à quarante-quatre. Bien entendu, il y a une certaine redondance entre les groupes. Toutefois, il est frappant de noter que sur ces quarante-quatre propositions, une seule correspond à un droit: avoir le droit de parler sans qu'on nous gêne. Pour ces enfants, les règles de l'école sont donc fortement 
ressenties comme une somme de contraintes contre lesquelles il n'aurait qu'un droit, celui de se taire. Ce qui laisse présager un travail en profondeur sur la formation du citoyen actif. Les règles soulignées (respecter les femmes à l'école et être autonome) sont, à mon avis, le résultat du travail incessant $d u$ titulaire de la classe qui insiste beaucoup sur l'autonomie des enfants et sur la place de la femme dans un milieu social où elle n'est pas toujours considérée comme l'égale de l'homme. »

La parole des élèves à l'école se situe immédiatement dans le registre du devoir et ne s'autorise pas à revendiquer ce qui est fondateur de la citoyenneté : des droits. Cette omniprésence de la règle nous a été confirmée par d'autres recherches. ${ }^{11}$

La citoyenneté est-elle un brevet ou un droit ? Les IO de 1985 citées par NV stipulent : «On naît citoyen. On devient un citoyen éclairé.» Une telle énormité introduit le germe d'une citoyenneté au mérite pour ceux qui ne peuvent plus bénéficier du droit du sol! Dans ce cas, il est risqué de commencer à jouer sur les mots, notamment ceux qui risquent d'entraîner une perte du sens traditionnel, national de la citoyenneté au profit d'un « droit du sang » qui a pour corollaire l'acquisition « au mérite » abandonné en 1998 par nos voisins allemands.

48 Peut-on préférer la formule de l'éducation de l'élève et du citoyen comme personne à l'éducation à la citoyenneté ? CP (p. 18) rencontre cette question dans son parcours et tente de lui donner une réponse en termes d'éducation:

"L'enfant est donc l'acteur essentiel de la construction de ses apprentissages : sollicité par l'enseignant, c'est à lui d'analyser la situation proposée, les difficultés rencontrées, et de rechercher les moyens nécessaires pour s'y adapter; mais dans ce type d'activité plus que dans les activités fondamentales, les problèmes posés le sont à l'ensemble du groupe; pourquoi ne pas parler alors de «constructivisme collectif ? "

Nous nous trouvons au bord de la notion ingénieuse de $\mathrm{J}$. Ardoino ${ }^{12}$ faisant remarquer que mieux que l'acteur, l'auteur est celui qui s'autorise à penser et à écrire par lui-même. La démocratie repose sur la notion de «négatricité ». Le fait de dire non, d'argumenter son refus, est un droit inaliénable en démocratie. Est-il si désirable dans la classe ? Les jeunes enseignants semblent le penser timidement. Qu'en est-il du discours constant de l'école en la matière?

50 Pendant la première journée de ce colloque, M. Laparra a insisté sur la nécessité d'apprendre l'école aux élèves, de distinguer soigneusement le privé du public. Il faut donc traiter la question éthique que posent ces mémoires : quel sens donne l'institution à sa mission d'éducation qui a succédé, en 1937, à celle d'instruction? L'école n'est-elle pas gênée dans son projet transmissif par le fonctionnement démocratique? Le savoir est-il objet de débat ou enjeu d'appropriation?

Le glissement du civique à la citoyenneté et l'instruction à l'éducation a bien un sens qu'il faut interroger. Les mémoires professionnels constituent un écrit précieux pour repérer et comprendre les évolutions actuelles et à venir des pratiques de classe : ce parcours rapide de cinq d'entre eux permet de repérer le besoin de clarification des concepts institutionnels autour de la citoyenneté et d'affirmer la nécessité d'une didactique de l'apprentissage citoyen qui est, par nature confié, à l'oral et à l'écrit réflexifs. 


\section{NOTES}

1. MEIR1EU P. : L'école ou la guerre civile. Paris, Plon, 1997, p. 11.

2. BAKHTINE M. : Le Marxisme et la philosophie du langage. Paris, Editions de Minuit,

3. Pour une étude du mémoire professionnel et de son rôle dans la formation des enseignants, on pourra se rapporter à - ÉTIENNE R. : «Le mémoire professionnel », in BOUVIER A. et OBIN J.-P. (éd.): La formation des enseignants sur le terrain. Paris, Hachette, 1998, p. 117-133

4. LE BOTER G. : De la compétence : essai sur un attracteur étrange. Paris, Les éditions d'organisation, 1994.

5. ÉTIENNE R. : «Une clef de voûte du mémoire professionnel », in Cahiers pédagogiques, № 373. 1999, p. 51-53.

6. Regroupement des diverses formes de mots possédant une racine commune pour aboutir à une statistique informatisée.

7. DEFRANCE B. : La planète lycéenne. Paris, Syros, 1996.

8. GAILLARD J. : Beau comme l'antique. Arles, Actes Sud, 1995.

9. CHAGNOLLAUD D. : Dictionnaire de la vie politique et sociale. Paris, Hatier, 1993.

10. DRORY D. : «Cris et châtiments», in Du bon usage de l'agressivité. Paris, Éd. conj. : De Boeck Belin, 1997.

11. ETIENNE R. : (à paraître) «Six élèves hésitant entre un rapport de soumission et un rapport de distanciation à la règle scolaire ", in Revue du CERFEE. Montpellier, Publications de l'Université Paul Valéry

12. ARDOINO J.: «Les enjeux de la décentralisation», in Cahiers pédagogiques, № 325. $1994 \mathrm{p}$. 14-15.

\section{RÉSUMÉS}

Il ne peut y avoir de citoyenneté possible sans la construction de la singularité de la pensée. L'école doit développer davantage des pratiques langagières réflexives, qui donnent aux élèves le pouvoir de penser et le pouvoir de réfléchir par eux-mêmes.

There is no citizenship possible without the development of individual thought. School must promote, more than it presently does, the language skills of reflective thinking, thereby empowering students to think and more importantly empowering them to think for themselves.

\section{INDEX}

Mots-clés : construction des connaissances, langage, pensée

Keywords : language, structuring of knowledge, thought 


\section{AUTEUR}

\section{DOMINIQUE BUCHETON}

Maître de Conférence, IUFM de Montpellier 